TAIWANESE JOURNAL OF MATHEMATICS

Vol. 9, No. 1, pp. 1-15, March 2005

This paper is available online at http://www.math.nthu.edu.tw/tjm/

\title{
STRONG CONVERGENCE THEOREMS FOR COMMUTATIVE NONEXPANSIVE SEMIGROUPS IN GENERAL BANACH SPACES
}

\author{
Hiromichi Miyake and Wataru Takahashi
}

\begin{abstract}
In this paper, we introduce an iteration scheme of Mann's type for general commutative nonexpansive semigroups and then obtain a strong convergence theorem in compact sets of general Banach spaces by using the theory of means of abstract semigroups. Using this result, we prove some strong convergence theorems in cases of discrete and one-parameter semigroups.
\end{abstract}

\section{INTRODUCTION}

In 1975, Baillon [3] originally studied the first nonlinear ergodic theorem in the framework of Hilbert spaces: Let $C$ be a closed and convex subset of a Hilbert space $H$ and let $T$ be a nonexpansive mapping of $C$ into itself. If the set $F(T)$ of fixed points of $T$ is nonempty, then for each $x \in C$, the Cesáro means

$$
S_{n}(x)=\frac{1}{n} \sum_{k=0}^{n-1} T^{k} x
$$

converge weakly to some $y \in F(T)$. In this case, putting $y=P x$ for each $x \in C$, $P$ is a nonexpansive retraction of $C$ onto $F(T)$ such that $P T=T P=P$ and $P x$ is contained in the closure of convex hull of $\left\{T^{n} x: n=1,2, \ldots\right\}$ for each $x \in C$. We call such a retraction "an ergodic retraction". Takahashi $[18,20]$ proved the existence of such a retraction for an amenable semigroup of nonexpansive mappings on a Hilbert space. Rodé [13] also found a sequence of means on a semigroup, generalizing the Cesàro means, and extended Baillon's theorem. These results were extended to a uniformly convex Banach space whose norm is Fréchet differentiable in the case of a commutative semigroup of nonexpansive mappings by Hirano, Kido and Takahashi [5]. In 1999, Lau, Shioji and Takahashi [8] extended Takahashi's result and Rodé's result to an amenable semigroup of nonexpansive mappings in the Banach space.

Received October 1, 2003; Accepted February 24, 2004.

Communicated by Mau-Hsiang Shih.

2000 Mathematics Subject Classification: Primary 49M05; Secondary 47H09, 47H20.

Key words and phrases: Strong convergence, Mann's type iteration, nonexpansive semigroups, invariant mean. 
On the other hand, in 1997, Shimizu and Takahashi [14] introduced the first iterative scheme for finding common fixed points of families of nonexpansive mappings and then many authors have studied such iterative schemes for families of various mappings (cf. [15,21]). For example, Atsushiba and Takahashi [1] studied an iteration scheme of Mann's type [6,7,11,12] for two commutative nonexpansive mappings $S$ and $T$ in a Banach space: $x_{0} \in C$ and

$$
x_{n+1}=\frac{\alpha_{n}}{n^{2}} \sum_{i=0}^{n-1} \sum_{j=0}^{n-1} S^{i} T^{j} x_{n}+\left(1-\alpha_{n}\right) x_{n}
$$

for each $n \geq 1$, where $\left\{\alpha_{n}\right\}$ is a sequence in [0,1]. Recently, Suzuki [16] and Suzuki and Takahashi [17] proved strong convergence theorems of the iteration scheme of Mann's type for two commutative nonexpansive mappings and one-parameter nonexpansive semigroups in compact sets of general Banach spaces, respectively.

In this paper, we introduce an iteration scheme of Mann's type for general commutative nonexpansive semigroups and then obtain a strong convergence theorem in compact sets of general Banach spaces by using the theory of means of abstract semigroups; for instance $[4,18,22]$. Using this result, we prove some strong convergence theorems in cases of discrete and one-parameter semigroups.

\section{Preliminaries}

Throughout this paper, we denote by $E$ a real Banach space with the topological dual $E^{*}$. Let $S$ be a semigroup. We denote by $l^{\infty}(S)$ the Banach space of all bounded real-valued functions on $S$ with supremum norm. For each $s \in S$, we define two operators $l(s)$ and $r(s)$ on $l^{\infty}(S)$ by $(l(s) f)(t)=f(s t)$ and $(r(s) f)(t)=$ $f(t s)$ for each $t \in S$ and $f \in l^{\infty}(S)$, respectively. Let $X$ be a subspace of $l^{\infty}(S)$ containing 1. An element $\mu$ of the topological dual $X^{*}$ of $X$ is said to be a mean on $X$ if $\|\mu\|=\mu(1)=1$. For $s \in S$, we can define a point evaluation $\delta_{s}$ by $\delta_{s}(f)=f(s)$ for each $f \in X$. A convex combination of point evaluations is called a finite mean on $X$. As is well known, $\mu$ is a mean on $X$ if and only if

$$
\inf _{s \in S} f(s) \leq \mu(f) \leq \sup _{s \in S} f(s)
$$

for each $f \in X$. Suppose that $l(s) X \in X$ and $r(s) X \in X$ for each $s \in S$. Then, a mean $\mu$ on $X$ is said to be left invariant (resp. right invariant) if $\mu(l(s) f)=\mu(f)$ (resp. $\mu(r(s) f)=\mu(f)$ ) for each $s \in S$ and $f \in X$. A mean $\mu$ on $X$ is said to be invariant if $\mu$ is both left and right invariant. $X$ is said to be amenable if there exists an invariant mean on $X$. For fixed point theorems for the semigroups, see [9]. We know from [4] that if $S$ is commutative, then $X$ is amenable. Let $\left\{\mu_{\alpha}\right\}$ be a net of means on $X$. Then $\left\{\mu_{\alpha}\right\}$ is said to be asymptotically invariant if for each $s \in S$, both $l(s)^{*} \mu_{\alpha}-\mu_{\alpha}$ and $r(s)^{*} \mu_{\alpha}-\mu_{\alpha}$ converge to 0 in the weak-star topology, 
where $l(s)^{*}$ and $r(s)^{*}$ are the adjoint operators of $l(s)$ and $r(s)$, respectively. Such nets were first studied by Day in [4]. We know that $X$ is amenable if and only if there exists an asymptotically invariant net of finite means on $X$ (cf. [4,22]).

Let $S$ be a commutative semigroup with identity. Then $S$ is a directed system when the binary relation $\leq$ on $S$ is defined by $s \leq t$ if and only if there exists $u \in S$ such that $t=u+s$. We know that if $S$ is a commutative semigroup and $\mu$ is an invariant mean on $X$, then

$$
\liminf _{s \in S} f(s) \leq \mu(f) \leq \limsup _{s \in S} f(s)
$$

for each $f \in X$; see $[19,22]$ for more details.

Let $T$ be a mapping of $C$ into itself. Then $T$ is said to be nonexpansive if $\|T x-T y\| \leq\|x-y\|$ for each $x, y \in C$. Let $S$ be a commutative semigroup with identity 0 and let $N \operatorname{Nonex}(C)$ be the set of all nonexpansive mappings of $C$ into itself. Then $\mathcal{S}=\{T(s): s \in S\}$ is called a nonexpansive semigroup on $C$ if $T(s) \in N$ onex $(C)$ for each $s \in S, T(0)=I$ and $T(s+t)=T(s) T(t)$ for each $s, t \in S$. We denote by $F(\mathcal{S})$ the set of all common fixed points of $\{T(s): s \in S\}$.

We denote by $l^{\infty}(S, E)$ the Banach space of all bounded mappings of $S$ into $E$ with supremum norm, and by $l_{c}^{\infty}(S, E)$ the subspace of all elements $f \in l^{\infty}(S, E)$ such that $f(S)=\{f(s): s \in S\}$ is a relatively weakly compact subset of $E$. Let $X$ be a subspace of $l^{\infty}(S)$ containing 1 such that $l(s) X \subset X$ for each $s \in S$, let $\mu$ be a mean on $X$ and let $\mathcal{S}=\{T(s): s \in S\}$ be a nonexpansive semigroup on $C$ such that $T(\cdot) x \in l_{c}^{\infty}(S, E)$ for some $x \in C$. If for each $x^{*} \in E^{*}$ the function $s \mapsto\left\langle T(s) x, x^{*}\right\rangle$ is contained in $X$, then it follows from the bipolar theorem that there exists a unique point $x_{0}$ of $E$ such that $\mu\left\langle T(\cdot) x, x^{*}\right\rangle=\left\langle x_{0}, x^{*}\right\rangle$ for each $x^{*} \in E^{*}$; see [18] and [5]. We denote such a point $x_{0}$ by $T_{\mu} x$. Note that $T_{\mu} x$ is contained in the closure of convex hull of $\{T(s) x: s \in S\}$; see [18] for more details.

To obtain the main result, we need the following lemma [16].

Lemma 1. Let $\left\{z_{n}\right\}$ and $\left\{w_{n}\right\}$ be bounded sequences in a Banach space $E$ and let $\left\{\alpha_{n}\right\}$ be a sequence in $[0,1]$ such that

$$
0<\liminf _{n \rightarrow \infty} \alpha_{n} \leq \limsup _{n \rightarrow \infty} \alpha_{n}<1 .
$$

Suppose that $z_{n+1}=\alpha_{n} w_{n}+\left(1-\alpha_{n}\right) z_{n}$ for each $n \geq 0$ and

$$
\limsup _{n \rightarrow \infty}\left(\left\|w_{n}-w_{n+k}\right\|-\left\|z_{n}-z_{n+k}\right\|\right) \leq 0
$$

for each $k \geq 0$. Then $\liminf \operatorname{in}_{n \rightarrow \infty}\left\|w_{n}-z_{n}\right\|=0$. 


\section{MaIn Result}

For proving our main theorem, we need the following proposition.

Proposition 1. Let $E$ be a Banach space, let $C$ be a compact convex subset of $E$, let $S$ be a commutative semigroup with identity, let $\mathcal{S}=\{T(s): s \in S\}$ be a nonexpansive semigroup on $C$, let $X$ be a subspace of $l^{\infty}(S)$ containing 1 such that $l(s) X \subset X$ for each $s \in S$ and the functions $s \mapsto\left\langle T(s) x, x^{*}\right\rangle$ and $s \mapsto\|T(s) x-y\|$ are contained in $X$ for each $x, y \in C$ and $x^{*} \in E^{*}$ and let $\left\{\mu_{n}\right\}$ be an asymptically invariant sequence of means on $X$. If $z \in C$ and $\liminf _{n \rightarrow \infty}\left\|T_{\mu_{n}} z-z\right\|=0$, then $z$ is a common fixed point of $\mathcal{S}$.

Proof. From liminf $\operatorname{in}_{n \rightarrow \infty}\left\|T_{\mu_{n}} z-z\right\|=0$, there exists a subsequence $\left\{T_{\mu_{n_{k}}} z\right\}$ of $\left\{T_{\mu_{n}} z\right\}$ such that $\left\{T_{\mu_{n_{k}}} z\right\}$ converges strongly to $z$. Since the set of means on $X$ is compact in the weak-star topology, there exists a subnet $\left\{\mu_{n_{k_{\alpha}}}: \alpha \in \Lambda\right\}$ of $\left\{\mu_{n_{k}}\right\}$ such that $\left\{\mu_{n_{k_{\alpha}}}\right\}$ converges to $\mu$ in the weak-star topology. Then, it follows that $\mu$ is an invariant mean on $X$. In fact, since $\left\{\mu_{n}\right\}$ is asymptotically invariant, for each $\epsilon>0, f \in X$ and $s \in S$, there exists $\alpha_{0} \in \Lambda$ such that

$$
\left|\mu_{n_{k_{\alpha}}}(f)-\mu_{n_{k_{\alpha}}}(l(s) f)\right| \leq \frac{\epsilon}{3}
$$

for each $\alpha \geq \alpha_{0}$. Since $\left\{\mu_{n_{k_{\alpha}}}\right\}$ converges to $\mu$ in the weak-star topology, we can choose $\beta \geq \alpha_{0}$ such that

$$
\left|\mu_{n_{k_{\beta}}}(f)-\mu(f)\right| \leq \frac{\epsilon}{3}
$$

and

$$
\left|\mu_{n_{k_{\beta}}}(l(s) f)-\mu(l(s) f)\right| \leq \frac{\epsilon}{3}
$$

Hence, we have

$$
\begin{aligned}
|\mu(f)-\mu(l(s) f)| & \leq\left|\mu(f)-\mu_{n_{k_{\beta}}}(f)\right|+\left|\mu_{n_{k_{\beta}}}(f)-\mu_{n_{k_{\beta}}}(l(s) f)\right| \\
& +\left|\mu_{n_{k_{\beta}}}(l(s) f)-\mu(l(s) f)\right| \\
& \leq \frac{\epsilon}{3}+\frac{\epsilon}{3}+\frac{\epsilon}{3}=\epsilon .
\end{aligned}
$$

Since $\epsilon>0$ is arbitrary, we have $\mu(f)=\mu(l(s) f)$ for each $f \in X$ and $s \in S$. This implies that $\mu$ is an invariant mean on $X$. It also follows that $\left\{T_{\mu_{n_{k_{\alpha}}}} z\right\}$ converges weakly to $\left\{T_{\mu} z\right\}$. In fact, for each $x^{*} \in E^{*}$, we have

$$
\left\langle T_{\mu_{n_{k_{\alpha}}}} z, x^{*}\right\rangle=\mu_{n_{k_{\alpha}}}\left\langle T(\cdot) z, x^{*}\right\rangle \rightarrow \mu\left\langle T(\cdot) z, x^{*}\right\rangle=\left\langle T_{\mu} z, x^{*}\right\rangle .
$$

On the other hand, since $\left\{T_{\mu_{n_{k}}} z\right\}$ converges strongly to $z$, we have $\left\langle z, x^{*}\right\rangle=$ $\left\langle T_{\mu} z, x^{*}\right\rangle$. This implies $z=T_{\mu} z$. 
Let $d=\mu\|T(\cdot) z-z\|$. Assume $d>0$. Since $\mu$ is an invariant mean on $X$, we have

$$
d=\mu\|T(\cdot) z-z\| \leq \limsup _{s \in S}\|T(s) z-z\| .
$$

Since $C$ is compact, there exists a cluster point $u_{1}$ of $\{T(s) z: s \in S\}$ in the norm topology such that $d \leq\left\|u_{1}-z\right\|$. Then, we have

$$
\begin{aligned}
d & \leq\left\|u_{1}-z\right\| \\
& =\left\|u_{1}-T_{\mu} z\right\| \\
& =\sup \left\{\left\langle u_{1}-T_{\mu} z, x^{*}\right\rangle: x^{*} \in E^{*},\left\|x^{*}\right\|=1\right\} \\
& =\sup \left\{\mu\left\langle u_{1}-T(\cdot) z, x^{*}\right\rangle: x^{*} \in E^{*},\left\|x^{*}\right\|=1\right\} \\
& \leq \sup \left\{\mu\left\|u_{1}-T(\cdot) z\right\|\left\|x^{*}\right\|: x^{*} \in E^{*},\left\|x^{*}\right\|=1\right\} \\
& =\mu\left\|u_{1}-T(\cdot) z\right\| .
\end{aligned}
$$

On the other hand, we have $\|T(t) z-z\| \leq d$ for each $t \in S$. In fact, since $\mu$ is an invariant mean on $X$, for each $t \in S$, we have

$$
\begin{aligned}
\|T(t) z-z\| & =\left\|T(t) z-T_{\mu} z\right\| \\
& \leq \mu\|T(t) z-T(\cdot) z\| .
\end{aligned}
$$

Putting $g(s)=\|T(t) z-T(s) z\|$ for each $s \in S$, we have $(l(t) g)(s)=\| T(t) z-$ $T(t+s) z \|$ and hence

$$
\begin{aligned}
\mu\|T(t) z-T(\cdot) z\| & =\mu(g)=\mu(l(t) g) \\
& =\mu(l(t)\|T(t) z-T(\cdot) z\|) \\
& =\mu\|T(t) z-T(t+\cdot) z\| \\
& \leq \mu\|z-T(\cdot) z\| \\
& =d .
\end{aligned}
$$

Let $\epsilon>0$ and $s \in S$. Since $u_{1}$ is a cluster point of $\{T(s) z: s \in S\}$, there exists $p \geq s$ such that $\left\|T(p) z-u_{1}\right\| \leq \epsilon / 2$. Then, for each $t \geq p$ with $t=p+w \in p+S$, we have

$$
\begin{aligned}
\left\|T(t) z-u_{1}\right\| & \leq\|T(t) z-T(p) z\|+\left\|T(p) z-u_{1}\right\| \\
& \leq\|T(w) z-z\|+\left\|T(p) z-u_{1}\right\| \\
& \leq d+\epsilon / 2 .
\end{aligned}
$$


Since $\mu$ is an invariant mean on $X$, we have

$$
\begin{aligned}
& \mu\left(\|T(p+\cdot) z-z\|+\left\|T(p+\cdot) z-u_{1}\right\|\right) \\
= & \mu\|T(p+\cdot) z-z\|+\mu\left\|T(p+\cdot) z-u_{1}\right\| \\
= & \mu\|T(\cdot) z-z\|+\mu\left\|T(\cdot) z-u_{1}\right\| \\
\geq & 2 d .
\end{aligned}
$$

Therefore, there exists $q \geq p$ such that

$$
\|T(q) z-z\|+\left\|T(q) z-u_{1}\right\| \geq 2 d-\epsilon / 2 .
$$

So, we have

$$
\begin{aligned}
\left\|T(q) z-u_{1}\right\| & \geq 2 d-\epsilon / 2-\|T(q) z-z\| \\
& \geq 2 d-\epsilon / 2-d \\
& \geq d-\epsilon / 2 \\
& >d-\epsilon
\end{aligned}
$$

and

$$
\begin{aligned}
\|T(q) z-z\| & \geq 2 d-\epsilon / 2-\left\|T(q) z-u_{1}\right\| \\
& \geq 2 d-\epsilon / 2-(d+\epsilon / 2) \\
& =d-\epsilon .
\end{aligned}
$$

Denote by $S(t)$ the closure of the set $\{T(s) z: t \leq s\}$ and set

$$
A=\bigcap_{t \in S} S(t)
$$

For $\epsilon>0$ and $u \in C$, we also put

$$
A(u, \epsilon)=\{x \in C:\|x-u\| \geq d-\epsilon\} .
$$

It follows from the above argument that the family of closed subsets consisting of $\{S(t): t \in S\}$ and $\left\{A(u, \epsilon): u \in\left\{z, u_{1}\right\}\right.$ and $\left.\epsilon>0\right\}$ has the finite intersection property. Since $C$ is compact, there exists a point $u_{2}$ of $A$ such that $d \leq\left\|u_{2}-u_{1}\right\|$ and $d \leq\left\|u_{2}-z\right\|$. We also have

$$
d \leq\left\|u_{2}-z\right\|=\left\|u_{2}-T_{\mu} z\right\| \leq \mu\left\|u_{2}-T(\cdot) z\right\| .
$$

Let $\epsilon>0$ and $s \in S$. Since $u_{1}$ and $u_{2}$ are a cluster point of $\{T(s) z: s \in S\}$, there exist $p_{1}, p_{2} \geq s$ such that $\left\|T\left(p_{1}\right) z-u_{1}\right\| \leq \epsilon / 3$ and $\left\|T\left(p_{2}\right) z-u_{2}\right\| \leq \epsilon / 3$. 
Then, for each $t \geq p_{1}+p_{2}$, we get $w_{1}, w_{2} \in S$ such that $t=w_{1}+p_{1}$ and $t=w_{2}+p_{2}$. So, we have, for each $i=1,2$,

$$
\begin{aligned}
\left\|T(t) z-u_{i}\right\| & \leq\left\|T(t) z-T\left(p_{i}\right) z\right\|+\left\|T\left(p_{i}\right) z-u_{i}\right\| \\
& \leq\left\|T\left(w_{i}\right) z-z\right\|+\left\|T\left(p_{i}\right) z-u_{i}\right\| \\
& \leq d+\epsilon / 3 .
\end{aligned}
$$

Choose $p \in S$ with $p \geq p_{1}+p_{2}$. Since $\mu$ is an invariant mean on $X$, we have

$$
\begin{aligned}
& \mu\left(\|T(p+\cdot) z-z\|+\left\|T(p+\cdot) z-u_{1}\right\|+\left\|T(p+\cdot) z-u_{2}\right\|\right) \\
= & \mu\|T(p+\cdot) z-z\|+\mu\left\|T(p+\cdot) z-u_{1}\right\|+\mu\left\|T(p+\cdot) z-u_{2}\right\| \\
= & \mu\|T(\cdot) z-z\|+\mu\left\|T(\cdot) z-u_{1}\right\|+\mu\left\|T(\cdot) z-u_{2}\right\| \\
\geq & 3 d .
\end{aligned}
$$

Therefore, there exists $q \geq p$ such that

$$
\|T(q) z-z\|+\left\|T(q) z-u_{1}\right\|+\left\|T(q) z-u_{2}\right\| \geq 3 d-\epsilon / 3 .
$$

So, we have $\|T(q) z-z\| \geq d-\epsilon$ and $\left\|T(q) z-u_{i}\right\| \geq d-\epsilon(i=1,2)$. Then, it follows that the family of closed subsets consisting of $\{S(t): t \in S\}$ and $\left\{A(u, \epsilon): u \in\left\{z, u_{1}, u_{2}\right\}\right.$ and $\left.\epsilon>0\right\}$ has the finite intersection property. Since $C$ is compact, there exists a point $u_{3}$ of $A$ such that $d \leq\left\|u_{i}-u_{j}\right\|$ and $d \leq\left\|u_{i}-z\right\|$ for each $i, j=1,2,3$ with $i \neq j$.

In the similar way, we can define inductively a sequence $\left\{u_{n}\right\}$ in $A$ such that for each $i, j$ with $i \neq j, d \leq\left\|u_{i}-u_{j}\right\|$ and for each $i \geq 1, d \leq\left\|u_{i}-z\right\|$. Since $C$ is compact, this is a contradiction. Hence, we have $d=0$.

Let $t \in S$. Then, we have, for each $s \in S$,

$$
\|T(t) z-z\| \leq\|T(t) z-T(t+s) z\|+\|T(t+s) z-z\| .
$$

Since $\mu$ is an invariant mean on $X$, we have

$$
\begin{aligned}
\|T(t) z-z\| & \leq \mu\|T(t) z-T(t+\cdot) z\|+\mu\|T(t+\cdot) z-z\| \\
& \leq \mu\|z-T(\cdot) z\|+\mu\|T(\cdot) z-z\| \\
& =d+d=0 .
\end{aligned}
$$

Then, $z$ is a common fixed point of $\mathcal{S}$. This completes the proof.

Now, using Lemma 1 and Proposition 1, we can prove a strong convergence theorem of Mann's type for general commutative nonexpansive semigroups on a compact convex subset of a Banach space. 
Theorem 1. Let $E$ be a Banach space, let $C$ be a compact convex subset of $E$, let $S$ be a commutative semigroup with identity, let $\mathcal{S}=\{T(s): s \in S\}$ be a nonexpansive semigroup on $C$, let $X$ be a subspace of $l^{\infty}(S)$ containing 1 such that $l(s) X \subset X$ for each $s \in S$ and the functions $s \mapsto\left\langle T(s) x, x^{*}\right\rangle$ and $s \mapsto\|T(s) x-y\|$ are contained in $X$ for each $x, y \in C$ and $x^{*} \in E^{*}$ and let $\left\{\mu_{n}\right\}$ be an asymptotically invariant sequence of means on $X$ such that $\lim _{n \rightarrow \infty}\left\|\mu_{n}-\mu_{n+1}\right\|=0$. Let $\left\{\alpha_{n}\right\}$ be a sequence in $[0,1]$ such that

$$
0<\liminf _{n \rightarrow \infty} \alpha_{n} \leq \limsup _{n \rightarrow \infty} \alpha_{n}<1 .
$$

Let $x_{0} \in C$ and let $\left\{x_{n}\right\}$ be the sequence defined by

$$
x_{n+1}=\alpha_{n} T_{\mu_{n}} x_{n}+\left(1-\alpha_{n}\right) x_{n}, \quad n=0,1,2, \ldots
$$

Then $\left\{x_{n}\right\}$ converges strongly to a common fixed point of $\mathcal{S}$.

Proof. For each $k, n \in \mathbb{N}$, we have

$$
\begin{aligned}
& \left\|T_{\mu_{n}} x_{n}-T_{\mu_{n+k}} x_{n+k}\right\|-\left\|x_{n}-x_{n+k}\right\| \\
\leq & \left\|T_{\mu_{n}} x_{n}-T_{\mu_{n}} x_{n+k}\right\|+\left\|T_{\mu_{n}} x_{n+k}-T_{\mu_{n+k}} x_{n+k}\right\|-\left\|x_{n}-x_{n+k}\right\| \\
\leq & \left\|x_{n}-x_{n+k}\right\|+\left\|T_{\mu_{n}} x_{n+k}-T_{\mu_{n+k}} x_{n+k}\right\|-\left\|x_{n}-x_{n+k}\right\| \\
= & \left\|T_{\mu_{n}} x_{n+k}-T_{\mu_{n+k}} x_{n+k}\right\| .
\end{aligned}
$$

Let $M=\sup _{x \in C}\|x\|$. Then, we have, for each $x^{*} \in E^{*}$ with $\left\|x^{*}\right\|=1$,

$$
\begin{aligned}
\left|\left\langle T_{\mu_{n}} x_{n+k}-T_{\mu_{n+k}} x_{n+k}, x^{*}\right\rangle\right| & =\left|\left\langle T_{\mu_{n}} x_{n+k}, x^{*}\right\rangle-\left\langle T_{\mu_{n+k}} x_{n+k}, x^{*}\right\rangle\right| \\
& =\left|\mu_{n}\left\langle T(\cdot) x_{n+k}, x^{*}\right\rangle-\mu_{n+k}\left\langle T(\cdot) x_{n+k}, x^{*}\right\rangle\right| \\
& =\left|\left(\mu_{n}-\mu_{n+k}\right)\left\langle T(\cdot) x_{n+k}, x^{*}\right\rangle\right| \\
& \leq\left\|\mu_{n}-\mu_{n+k}\right\| \sup _{s \in S}\left|\left\langle T(s) x_{n+k}, x^{*}\right\rangle\right| \\
& \leq\left\|\mu_{n}-\mu_{n+k}\right\| \sup _{s \in S}\left\|T(s) x_{n+k}\right\|\left\|x^{*}\right\| \\
& \leq M\left\|\mu_{n}-\mu_{n+k}\right\|
\end{aligned}
$$

and hence

$$
\left\|T_{\mu_{n}} x_{n+k}-T_{\mu_{n+k}} x_{n+k}\right\| \leq M\left\|\mu_{n}-\mu_{n+k}\right\| .
$$

From $\lim _{n \rightarrow \infty}\left\|\mu_{n}-\mu_{n+1}\right\|=0$, we have

$$
\begin{aligned}
& \lim \sup _{n \rightarrow \infty}\left(\left\|T_{\mu_{n}} x_{n}-T_{\mu_{n+k}} x_{n+k}\right\|-\left\|x_{n}-x_{n+k}\right\|\right) \\
\leq & \lim _{n \rightarrow \infty}\left\|T_{\mu_{n}} x_{n+k}-T_{\mu_{n+k}} x_{n+k}\right\| \\
= & 0 .
\end{aligned}
$$


Hence, it follows from Lemma 1 that $\liminf _{n \rightarrow \infty}\left\|T_{\mu_{n}} x_{n}-x_{n}\right\|=0$. Thus, there exists a subsequence $\left\{x_{n_{k}}\right\}$ of $\left\{x_{n}\right\}$ such that $\lim _{k \rightarrow \infty}\left\|T_{\mu_{n_{k}}} x_{n_{k}}-x_{n_{k}}\right\|=0$. Further, since $C$ is compact, there exist a subsequence $\left\{x_{n_{k_{i}}}\right\}$ of $\left\{x_{n_{k}}\right\}$ and a point $z$ of $C$ such that $\left\{x_{n_{k_{i}}}\right\}$ converges strongly to $z$. Without loss of generality, we assume that there exist a subsequence $\left\{x_{n_{k}}\right\}$ of $\left\{x_{n}\right\}$ and a point $z \in C$ such that $\left\{x_{n_{k}}\right\}$ converges strongly to $z$ and $\lim _{k \rightarrow \infty}\left\|T_{\mu_{n_{k}}} x_{n_{k}}-x_{n_{k}}\right\|=0$. Then since

$$
\begin{aligned}
\left\|T_{\mu_{n_{k}}} z-z\right\| \leq & \leq\left\|T_{\mu_{n_{k}}} z-T_{\mu_{n_{k}}} x_{n_{k}}\right\|+\left\|T_{\mu_{n_{k}}} x_{n_{k}}-x_{n_{k}}\right\|+\left\|x_{n_{k}}-z\right\| \\
& \leq 2\left\|x_{n_{k}}-z\right\|+\left\|T_{\mu_{n_{k}}} x_{n_{k}}-x_{n_{k}}\right\|,
\end{aligned}
$$

we have $\lim _{k \rightarrow \infty}\left\|T_{\mu_{n_{k}}} z-z\right\|=0$ and hence $\liminf \operatorname{in}_{n \rightarrow \infty}\left\|T_{\mu_{n}} z-z\right\|=0$. Therefore, it follows from Proposition 1 that $z$ is a common fixed point of $\mathcal{S}$. Since

$$
\begin{aligned}
\left\|x_{n+1}-z\right\| & \leq \alpha_{n}\left\|T_{\mu_{n}} x_{n}-z\right\|+\left(1-\alpha_{n}\right)\left\|x_{n}-z\right\| \\
& \leq \alpha_{n}\left\|x_{n}-z\right\|+\left(1-\alpha_{n}\right)\left\|x_{n}-z\right\| \\
& =\left\|x_{n}-z\right\|,
\end{aligned}
$$

the $\lim _{n \rightarrow \infty}\left\|x_{n}-z\right\|$ exists. Thus, we have

$$
\lim _{n \rightarrow \infty}\left\|x_{n}-z\right\|=\lim _{k \rightarrow \infty}\left\|x_{n_{k}}-z\right\|=0 .
$$

This completes the proof.

\section{Some Strong Convergence Theorems}

In this section, applying the generalized strong convergence theorem of Mann's type for nonexpansive semigroups in Section 3, we obtain some strong convergence theorems in a Banach space. We denote by $\mathbb{N}, \mathbb{N}_{+}$and $\mathbb{R}_{+}$the set of all nonnegative integers, the set of all positive integers and the set of all nonnegative real numbers, respectively.

Theorem 2 ([16]). Let $E$ be a Banach space, let $C$ be a compact convex subset of $E$, let $S$ and $T$ be nonexpansive mappings of $C$ into itself with $S T=T S$. Let $x_{0} \in C$ and let $\left\{x_{n}\right\}$ be the sequence defined by

$$
x_{n+1}=\frac{\alpha_{n}}{n^{2}} \sum_{i=0}^{n-1} \sum_{j=0}^{n-1} S^{i} T^{j} x_{n}+\left(1-\alpha_{n}\right) x_{n}
$$

for each $n \in \mathbb{N}$, where $\left\{\alpha_{n}\right\}$ is a sequence in $[0,1]$ such that

$$
0<\liminf _{n \rightarrow \infty} \alpha_{n} \leq \limsup _{n \rightarrow \infty} \alpha_{n}<1 .
$$


Then $\left\{x_{n}\right\}$ converges strongly to a common fixed point of $S$ and $T$.

Proof. Let $T(k)=S^{i} T^{j}$ for each $k=(i, j)$ in $\mathbb{N}^{2}$. Since $S^{i}$ and $T^{j}$ are nonexpansive for each $i, j \in \mathbb{N}$ and $S T=T S,\left\{T(k): k \in \mathbb{N}^{2}\right\}$ is a nonexpansive semigroup on $C$. For each $n \in \mathbb{N}_{+}$, let us define

$$
\mu_{n}(x)=\frac{1}{n^{2}} \sum_{i=0}^{n-1} \sum_{j=0}^{n-1} x(i, j)
$$

for each $x \in l^{\infty}\left(\mathbb{N}^{2}\right)$. Then, $\left\{\mu_{n}\right\}$ is an asymptotically invariant sequence of means; for more details, see [22]. Next, for each $x \in C, x^{*} \in E^{*}, k \in \mathbb{N}^{2}$ and $n \in \mathbb{N}_{+}$, we have

$$
\begin{aligned}
\mu_{n}\left\langle T(k) x, x^{*}\right\rangle & =\frac{1}{n^{2}} \sum_{i=0}^{n-1} \sum_{j=0}^{n-1}\left\langle S^{i} T^{j} x, x^{*}\right\rangle \\
& =\left\langle\frac{1}{n^{2}} \sum_{i=0}^{n-1} \sum_{j=0}^{n-1} S^{i} T^{j} x, x^{*}\right\rangle .
\end{aligned}
$$

Then, we have

$$
T_{\mu_{n}} x=\frac{1}{n^{2}} \sum_{i=0}^{n-1} \sum_{j=0}^{n-1} S^{i} T^{j} x
$$

for each $n \in \mathbb{N}_{+}$.

On the other hand, for each $x \in l^{\infty}\left(\mathbb{N}^{2}\right)$ with $\|x\|=1$, we have

$$
\begin{aligned}
& \left|\mu_{n+1}(x)-\mu_{n}(x)\right| \\
= & \left|\frac{1}{(n+1)^{2}} \sum_{i=0}^{n} \sum_{j=0}^{n} x(i, j)-\frac{1}{n^{2}} \sum_{i=0}^{n-1} \sum_{j=0}^{n-1} x(i, j)\right| \\
\leq & \left|\frac{1}{(n+1)^{2}} \sum_{i=0}^{n} \sum_{j=0}^{n} x(i, j)-\frac{1}{(n+1)^{2}} \sum_{i=0}^{n-1} \sum_{j=0}^{n-1} x(i, j)\right| \\
& +\left|\frac{1}{(n+1)^{2}} \sum_{i=0}^{n-1} \sum_{j=0}^{n-1} x(i, j)-\frac{1}{n^{2}} \sum_{i=0}^{n-1} \sum_{j=0}^{n-1} x(i, j)\right| \\
\leq & \frac{1}{(n+1)^{2}}\left|\sum_{\substack{i=n \\
\text { or } j=n}} x(i, j)\right|+\left(\frac{1}{n^{2}}-\frac{1}{(n+1)^{2}}\right)\left|\sum_{i=0}^{n-1} \sum_{j=0}^{n-1} x(i, j)\right| \\
\leq & \frac{1}{(n+1)^{2}} \sum_{\substack{i=n \\
\text { or } j=n}}|x(i, j)|+\left(\frac{1}{n^{2}}-\frac{1}{(n+1)^{2}}\right) \sum_{i=0}^{n-1} \sum_{j=0}^{n-1}|x(i, j)|
\end{aligned}
$$




$$
\leq \frac{2 n}{(n+1)^{2}}+\left(\frac{1}{n^{2}}-\frac{1}{(n+1)^{2}}\right) n^{2}
$$

and hence

$$
\left\|\mu_{n+1}-\mu_{n}\right\| \leq \frac{2 n}{(n+1)^{2}}+\left(\frac{1}{n^{2}}-\frac{1}{(n+1)^{2}}\right) n^{2}
$$

for each $n \in \mathbb{N}_{+}$. Thus, we have $\lim _{n \rightarrow \infty}\left\|\mu_{n+1}-\mu_{n}\right\|=0$.

Therefore, it follows from Theorem 1 that $\left\{x_{n}\right\}$ converges strongly to a common fixed point of $S$ and $T$. This completes the proof.

Let $Q=\left\{q_{n, m}\right\}_{n, m \in}$ be a matrix satisfying the following conditions:

(a) $\sup _{n \geq 0} \sum_{m=0}^{\infty}\left|q_{n, m}\right|<\infty$;

(b) $\sum_{m=0}^{\infty} q_{n, m}=1$ for each $n \in \mathbb{N}$;

(c) $\lim _{n \rightarrow \infty} \sum_{m=0}^{\infty}\left|q_{n, m+1}-q_{n, m}\right|=0$;

(d) $\lim _{n \rightarrow \infty} \sum_{m=0}^{\infty}\left|q_{n+1, m}-q_{n, m}\right|=0$.

Then, $Q$ is called a weighted mean matrix. Note that a weighted mean matrix $Q$ is a special case of strongly regular matrix; see [10]. If $Q$ is a strongly regular matrix, then for each $m \in \mathbb{N}$, we have $\left|q_{n, m}\right| \rightarrow 0$ as $n \rightarrow \infty$; see [5].

Theorem 3. Let $E$ be a Banach space, let $C$ be a compact convex subset of $E$, let $T$ be a nonexpansive mapping of $C$ into itself and let $Q=\left\{q_{n, m}\right\}$ be a weighted mean matrix. Let $x_{0} \in C$ and let the sequence $\left\{x_{n}\right\}$ be define by

$$
x_{n+1}=\alpha_{n} \sum_{m=0}^{\infty} q_{n, m} T^{m} x_{n}+\left(1-\alpha_{n}\right) x_{n}
$$

for each $n \in \mathbb{N}$, where $\left\{\alpha_{n}\right\}$ is a sequence in $[0,1]$ such that

$$
0<\liminf _{n \rightarrow \infty} \alpha_{n} \leq \limsup _{n \rightarrow \infty} \alpha_{n}<1 .
$$

Then $\left\{x_{n}\right\}$ converges strongly to a fixed point of $T$.

Proof. For each $n \in \mathbb{N}$, let us define

$$
\mu_{n}(x)=\sum_{m=0}^{\infty} q_{n, m} x_{m}
$$

for each $x=\left(x_{0}, x_{1}, x_{2}, \ldots\right) \in l^{\infty}(\mathbb{N})$. Then, $\left\{\mu_{n}\right\}$ is an asymptotically invariant sequence of means; for more details, see [22]. Next, for each $x \in C, x^{*} \in E^{*}$ and $n \in \mathbb{N}$, we have 


$$
\begin{aligned}
\mu_{n}\left\langle T^{m} x, y\right\rangle & =\sum_{m=0}^{\infty} q_{n, m}\left\langle T^{m} x, y\right\rangle \\
& =\left\langle\sum_{m=0}^{\infty} q_{n, m} T^{m} x, y\right\rangle .
\end{aligned}
$$

Then, we have $T_{\mu_{n}} x=\sum_{m=0}^{\infty} q_{n, m} T^{m} x$.

On the other hand, for each $x \in l^{\infty}(\mathbb{N})$ with $\|x\|=1$, we have

$$
\begin{aligned}
\left|\mu_{n}(x)-\mu_{n+1}(x)\right| & =\left|\sum_{m=0}^{\infty} q_{n, m} x_{m}-\sum_{m=0}^{\infty} q_{n+1, m} x_{m}\right| \\
& =\left|\sum_{m=0}^{\infty}\left(q_{n, m}-q_{n+1, m}\right) x_{m}\right| \\
& \leq \sum_{m=0}^{\infty}\left|q_{n, m}-q_{n+1, m}\right|\left|x_{m}\right| \\
& \leq \sum_{m=0}^{\infty}\left|q_{n, m}-q_{n+1, m}\right|
\end{aligned}
$$

and hence we have $\left\|\mu_{n}-\mu_{n+1}\right\| \leq \sum_{m=0}^{\infty}\left|q_{n, m}-q_{n+1, m}\right|$ for each $n \in \mathbb{N}$. Thus, we have $\lim _{n \rightarrow \infty}\left\|\mu_{n+1}-\mu_{n}\right\|=0$.

Therefore, it follows from Theorem 1 that $\left\{x_{n}\right\}$ converges strongly to a fixed point of $T$. This completes the proof.

Theorem 4 ([17]). Let $E$ be a Banach space, let $C$ be a compact convex subset of $E$ and let $\mathcal{S}=\left\{T(t): t \in \mathbb{R}_{+}\right\}$be a strongly continuous nonexpansive semigroup on $C$. Let $x_{0} \in C$ and let $\left\{x_{n}\right\}$ be the sequence define by

$$
x_{n+1}=\frac{\alpha_{n}}{t_{n}} \int_{0}^{t_{n}} T(s) x_{n} d s+\left(1-\alpha_{n}\right) x_{n}
$$

for each $n \in \mathbb{N}$, where $\left\{\alpha_{n}\right\}$ is a sequence in $[0,1]$ such that

$$
0<\liminf _{n \rightarrow \infty} \alpha_{n} \leq \limsup _{n \rightarrow \infty} \alpha_{n}<1
$$

and $\left\{t_{n}\right\}$ is an increasing sequence in $(0, \infty]$ such that $\lim _{n \rightarrow \infty} t_{n}=\infty$ and $\lim _{n \rightarrow \infty} t_{n} / t_{n+1}=1$. Then $\left\{x_{n}\right\}$ converges strongly to a common fixed point of $\mathcal{S}$.

Proof. For $n \in \mathbb{N}$, let us define

$$
\mu_{n}(f)=\frac{1}{t_{n}} \int_{0}^{t_{n}} f(t) d t
$$


for each $f \in C\left(\mathbb{R}_{+}\right)$, where $C\left(\mathbb{R}_{+}\right)$denote the space of all real-valued bounded continuous functions on $\mathbb{R}_{+}$with supremum norm. Then, $\left\{\mu_{n}\right\}$ is an asymptotically invariant sequence of means; for more details, see [22]. Further, for each $x \in C$ and $x^{*} \in E^{*}$, we have

$$
\begin{aligned}
\mu_{n}\left\langle T(\cdot) x, x^{*}\right\rangle & =\frac{1}{t_{n}} \int_{0}^{t_{n}}\left\langle T(s) x, x^{*}\right\rangle d s \\
& =\left\langle\frac{1}{t_{n}} \int_{0}^{t_{n}} T(s) x d s, x^{*}\right\rangle .
\end{aligned}
$$

Then, we have

$$
T_{\mu_{n}} x=\frac{1}{t_{n}} \int_{0}^{t_{n}} T(s) x d s .
$$

On the other hand, for each $f \in C\left(\mathbb{R}_{+}\right)$with $\|f\|=1$, we have

$$
\begin{aligned}
\left|\mu_{n+1}(f)-\mu_{n}(f)\right|= & \left|\frac{1}{t_{n+1}} \int_{0}^{t_{n+1}} f(s) d s-\frac{1}{t_{n}} \int_{0}^{t_{n}} f(s) d s\right| \\
\leq & \left|\frac{1}{t_{n+1}} \int_{0}^{t_{n+1}} f(s) d s-\frac{1}{t_{n+1}} \int_{0}^{t_{n}} f(s) d s\right| \\
& +\left|\frac{1}{t_{n+1}} \int_{0}^{t_{n}} f(s) d s-\frac{1}{t_{n}} \int_{0}^{t_{n}} f(s) d s\right| \\
= & \frac{1}{t_{n+1}}\left|\int_{t_{n}}^{t_{n+1}} f(s) d s\right|+\left(\frac{1}{t_{n}}-\frac{1}{t_{n+1}}\right)\left|\int_{0}^{t_{n}} f(s) d s\right| \\
\leq & \frac{1}{t_{n+1}} \int_{t_{n}}^{t_{n+1}}|f(s)| d s+\left(\frac{1}{t_{n}}-\frac{1}{t_{n+1}}\right) \int_{0}^{t_{n}}|f(s)| d s \\
\leq & \frac{t_{n+1}-t_{n}}{t_{n+1}}+\left(\frac{1}{t_{n}}-\frac{1}{t_{n+1}}\right) t_{n} \\
= & 2-2 \frac{t_{n}}{t_{n+1}}
\end{aligned}
$$

and hence $\left\|\mu_{n+1}-\mu_{n}\right\| \leq 2-2 t_{n} / t_{n+1}$ for each $n \in \mathbb{N}$. Thus, we have $\lim _{n \rightarrow \infty}\left\|\mu_{n+1}-\mu_{n}\right\|=0$.

Therefore, it follows from Theorem 1 that $\left\{x_{n}\right\}$ converges strongly to a common fixed point of $\mathcal{S}$. This completes the proof.

\section{REFERENCES}

1. S. Atsushiba, and W. Takahashi, Approximating common fixed points of two nonexpansive mappings in Banach spaces, Bull. Austral. Math. Soc., 57 (1998), 117-127. 
2. S. Atsushiba, and W. Takahashi, Strong convergence theorems for one-parameter nonexpansive semigroups with compact domains, Fixed Point Theory and Applications, Volume 3 (Y. J. Cho, J. K. Kim and S. M. Kang Eds.), pp. 15-31, Nova Science Publishers, New York, 2002.

3. J. B. Baillon, Un théorème de type ergodique pour les contractions non linéaires dans un espace de Hilbert, C. R. Acad. Sci. Paris Sér. A-B, 280 (1975), 1511-1514.

4. M. M. Day, Amenable semigroup, Illinois J. Math., 1 (1957), 509-544.

5. N. Hirano, K. Kido, and W. Takahashi, Nonexpansive retractions and nonlinear ergodic theorems in Banach spaces, Nonlinear Anal., 12 (1988), 1269-1281.

6. S. Ishikawa, Fixed points and iteratoin of a nonexpansive mapping in a Banach space, Proc. Amer. Math. Soc., 59 (1976), 65-71.

7. M. Krasnosel'ski, Two remarks on the method of successive approximations, Uspehi Mat. Nauk, 10 (1955), 123-127.

8. A. T .Lau, N. Shioji, and W. Takahashi, Existence of nonexpansive retractions for amenable semigroups of nonexpansive mappings and nonlinear ergodic theorems in Banach spaces, J. Funct. Anal., 161 (1999), 62-75.

9. A. T. Lau, and W. Takahashi, Invariant submeans and semigroups of nonexpansive mappings on Banach spaces with normal structure, J. Funct. Anal., 142 (1996), 79-88.

10. G. G. Lorentz, A contribution to the theory of divergent series, Acta Math., 80 (1948), 167-190.

11. W. Mann, Mean value methods in iteration, Proc. Amer. Math. Soc., 4 (1953), 506-510.

12. S. Reich, Weak convergence theorems for nonexpansive mappings, J. Math. Anal. Appl., 67 (1979), 274-276.

13. G. Rodé, An ergodic theorem for semigroups of nonexpansive mappings in a Hilbert space, J. Math. Anal. Appl., 85 (1982), 172-178.

14. T. Shimizu, and W. Takahashi, Strong convergen to common fixed points of families of nonexpansive mappings, J. Math. Anal. Appl., 211 (1997), 71-83.

15. N. Shioji, and W. Takahashi, Strong convergence theorems for asymptotically nonexpansive semigroups in Banach spaces, J. Nonlinear Convex Anal., 1 (2000), 73-87.

16. T. Suzuki, Strong convergence theorem to common fixed points of two nonexpansive mappings in general Banach spaces, J. Nonlinear Convex Anal., 3 (2002), 381-391.

17. T. Suzuki, and W. Takahashi, Strong convergence of Mann's type sequences for one-parameter nonexpansive semigroups in general Banach spaces, to appear in $J$. Nonlinear Convex Anal.

18. W. Takahashi, A nonlinear ergodic theorem for an amenable semigroup of nonexpansive mappings in a Hilbert space, Proc. Amer. Math. Soc., 81 (1981), 253-256. 
19. W. Takahashi, Fixed point theorems for families of nonexpansive mappings on unbounded sets, J. Math. Soc. Japan, 36 (1984), 543-553.

20. W. Takahashi, Fixed point theorem and nonlinear ergodic theorem for nonexpansive semigroups without convexity, Canad. J. Math., 44 (1992), 880-887.

21. W. Takahashi, Iterative methods for approximation of fixed points and their applications, J. Oper. Res. Soc. Japan, 43 (2000), 87-108.

22. W. Takahashi, Nonlinear Functional Analysis, Yokohama Publishers, Yokohama, 2000 .

Hiromichi Miyake and Wataru Takahashi

Department of Mathematical and Computing Sciences,

Tokyo Institute of Technology,

Ohokayama, Meguro-ku,

Tokyo 152, Japan

E-mail: miyake@is.titech.ac.jp and wataru@is.titech.ac.jp 